\title{
The incidence of preoperative anemia in patients undergoing cardiac surgery
}

Euroanaesthesia 2018 :

In a multicenter cohort study, the incidence of preoperative anemia in cardiac surgery patients was found to be $26 \%$ on average, up to a maximum of $30 \%$

(I). In another study of elective vascular surgery patients, the preoperative anemia incidence was $47 \%$ and the risk of mortality and cardiac events in these patients was $\mathbf{2 . 4 \%}$ (2). Previous studies in cardiac surgical patients have been limited, especially with small sample numbers $(3,4)$. Our hypothesis in this study was that the incidence of preoperative anemia may be high in patients undergoing cardiac surgery and this may be related to mortality and perioperative complications. We believe that the awareness of preoperative anemia can be increased according to the results of this study,

that elective patients can undergo surgery with

preoperative anemia treatment. Thus consequently

blood and blood product transfusion requirement and related complications can be reduced.

Following Ethics Committee approval, adult patients undergoing bypass surgery due to ischemic heart disease between 2014 and 2017 were evaluated retrospectively. Patient demographics, cardiac reserves, preoperative and postoperative Htc values, transfusion volume of blood and blood products, complications and mortality rates were recorded. Other laboratory tests, type of surgery, re-exploration frequency, intensive care and hospital stay were recorded. According to the World Health Organization, for women to have anemia, the Hb value should be $<12.0 \mathrm{~g} / \mathrm{dL}$, and for men, $<13.0 \mathrm{~g} / \mathrm{dL}$ (5). Preoperative anemia was diagnosed based on these subvalues in our study.

able 1: Patient demographics, transf rates and complications

\begin{tabular}{|c|c|c|c|c|c|c|c|}
\hline \multirow{2}{*}{ Age (years) } & & \multicolumn{2}{|c|}{$\min -\max$} & \multirow{2}{*}{$\frac{\text { median }}{64,0}$} & \multicolumn{3}{|c|}{ meantsd/n(\%) } \\
\hline & & 37,0 & 89,0 & & 64,3 & \pm & 10,0 \\
\hline \multirow{2}{*}{ Gender } & Female & & & & 57 & & $17,1 \%$ \\
\hline & Male & & & & 276 & & $82,9 \%$ \\
\hline & & 22,5 & 31,6 & 26,0 & 26,6 & \pm & 1,9 \\
\hline Duration of surgery (min) & & 25,0 & 300,0 & 210,0 & 217,1 & \pm & 35,0 \\
\hline CPB time & & 25,0 & 220,0 & 80,0 & 84,7 & \pm & 21,8 \\
\hline CX time & & 10,0 & $-135,0$ & 65,0 & 64,8 & \pm & 18,6 \\
\hline Drainage in ICU (mL) & & 150,0 & 2500,0 & 450,0 & 446,1 & \pm & 254,7 \\
\hline \multirow{2}{*}{ Stax in ICU } & 1 day & & & & 271 & & $81,4 \%$ \\
\hline & $\geq 2$ days & & & & 62 & & $18,6 \%$ \\
\hline \multirow{2}{*}{ Stax in hospital } & 7 days & & & & 259 & & $77,8 \%$ \\
\hline & 8 days & & & & 72 & & $21,6 \%$ \\
\hline \multirow{2}{*}{ Mortality rate } & $(-)$ & & & & 331 & & $99,4 \%$ \\
\hline & $(+)$ & & & & 2 & & $0,6 \%$ \\
\hline RBC & & 1,0 & 10,0 & 2,0 & 2,9 & \pm & 1,7 \\
\hline \multirow{2}{*}{ RBC } & Not transfused & & & & 43 & & $12,9 \%$ \\
\hline & Transfused & & & & 290 & & $87,1 \%$ \\
\hline FFP & & 1,0 & 56,0 & 2,0 & 3,7 & \pm & 5,9 \\
\hline \multirow{2}{*}{ FFP } & Not transfused & & & & 166 & & $49,8 \%$ \\
\hline & Transfused & & & & 167 & & $50,2 \%$ \\
\hline Platelet & & 2,0 & 124,0 & 16,0 & 29,3 & \pm & 30,0 \\
\hline \multirow{2}{*}{ Platelet } & Not transfused & & & & 285 & & $85,6 \%$ \\
\hline & Transfused & & & & 48 & & $14,4 \%$ \\
\hline \multirow[b]{2}{*}{ Cryopresipitate } & Not transfused & & & & 327 & & $98,2 \%$ \\
\hline & Transfused & & & & 6 & & $1,8 \%$ \\
\hline \multirow{3}{*}{$\begin{array}{l}\text { Perioperative bleeding (mL) } \\
\text { EF (\%) }\end{array}$} & & 100,0 & 3400,0 & 600,0 & 715,7 & \pm & 452,3 \\
\hline & & 38,0 & - 60,0 & 50,0 & 50,7 & \pm & \\
\hline & $(-)$ & & & & 333 & & $100,0 \%$ \\
\hline Postoperative DVT & $(+)$ & & & & 0 & & $0,0 \%$ \\
\hline \multirow{2}{*}{ Postoperative stroke } & $(-)$ & & & & 333 & & $100,0 \%$ \\
\hline & $(+)$ & & & & 0 & & $0,0 \%$ \\
\hline \multirow{2}{*}{ Reexploration } & $(-)$ & & & & 324 & & $97,3 \%$ \\
\hline & (+) & & & & 9 & & $2,7 \%$ \\
\hline
\end{tabular}

In total the data of 333 patients were evaluated. The incidence of anemia was $43 \% .265$ patients received RBC transfusions $(\mathbf{7 9 . 6 \% )}$ and $75 \%$ of blood transfusions were found in the anemic group compared to the RBC transfusions in the non anemic group and $89 \%$ in the anemia group ( $p=0.005$, Table 1,2$)$. Perioperative bleeding ranged from $100 \mathrm{~mL}$ to $3400 \mathrm{~mL}$ with a mean of $715.7 \pm 452.3 \mathrm{~mL}$, and the mean amount of bleeding was significantly higher in the anemic group than in the nonanemic group $(p=0.000)$. Postoperative $\mathrm{Hb}$, Htc and platelet values decreased at the end of the surgery compared to preoperative values in all patients.

Statistically significant increase in creatinine values was detected ( $p<0.005$, Table 3). Nine patients were reexplored. The use of FFP in the anemic group was higher than in nonanemic patients $(60.0 \%, 45.9 \%$, respectively, $p=0.019)$. There was no difference between the two groups in terms of mortality, hospital and ICU stay. There was no difference in terms of drainage and hospital mortality in ICU.

ble 2: Outcomes in anemic vs non-artente patients

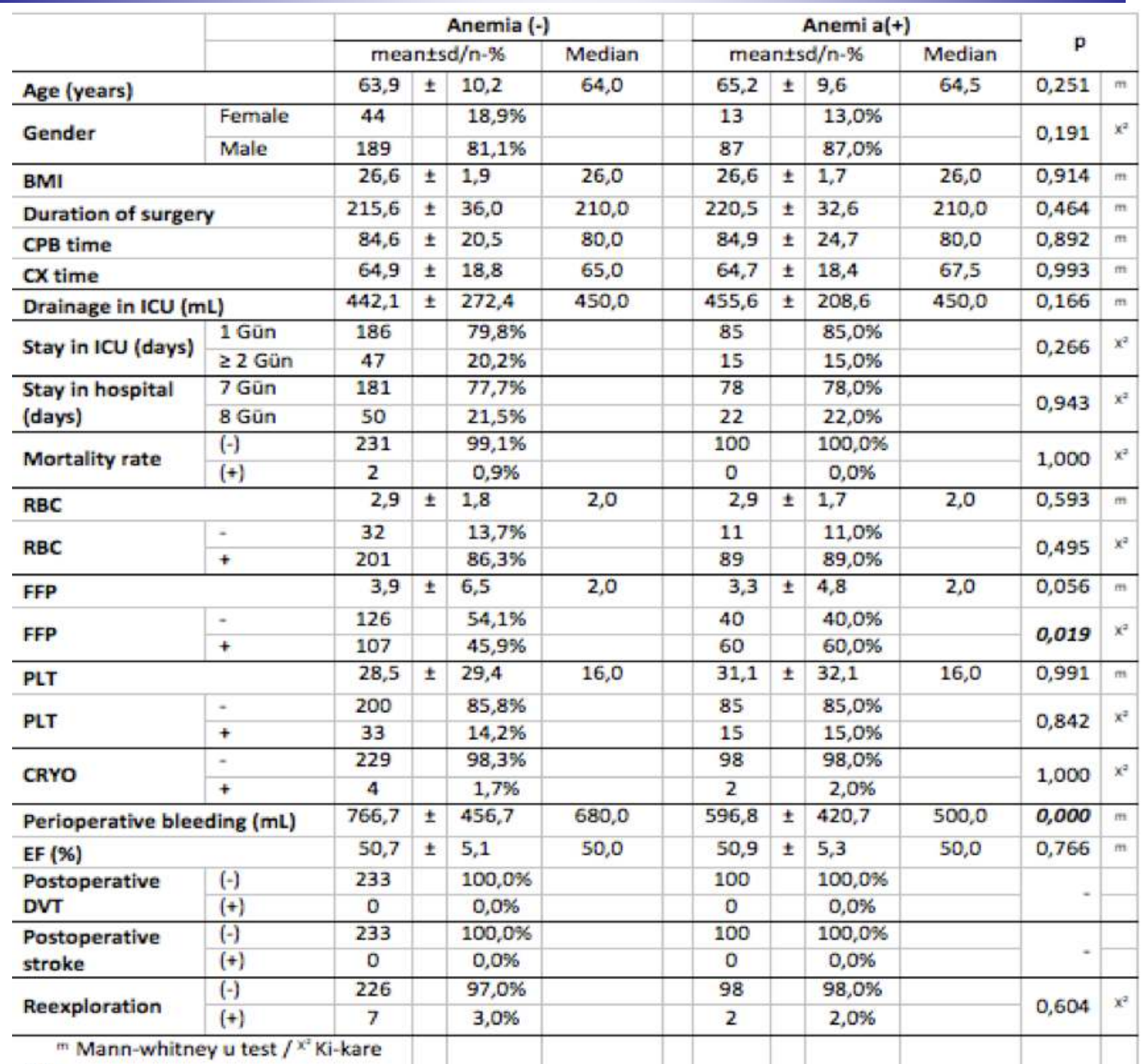

m Mann-whitney u test $/ \mathrm{x}^{\mathrm{T}}$ Ki-kare

Despite the published guidelines, the incidence of anemia in the preoperative period was high in our study similar to the literature. Although our study did not lead to an increase in complications statistically, the risks associated with $\mathrm{RBC}$ and FFP transfusion in the perioperative period exsts. In order to reduce this, anemia treatment should be included in the preoperative preparation of cardiac patients. 